\title{
Magnified endoscopy with texture and color enhanced imaging with indigo carmine for superficial nonampullary duodenal tumor: A pilot study
}

Kenichiro Okimoto ( $\nabla$ okimoto-k@chiba-u.jp )

Chiba University

Tomoaki Matsumura

Chiba University

Daisuke Maruoka

Chiba University

Akane Kurosugi

Chiba University

Wataru Shiratori

Chiba University

Ariki Nagashima

Chiba University

Tsubasa Ishikawa

Chiba University

Tatsuya Kaneko

Chiba University

Kengo Kanayama

Chiba University

Naoki Akizue

Chiba University

Yuki Ohta

Chiba University

Takashi Taida

Chiba University

Keiko Saito

Chiba University

Jun Kato

Chiba University

Naoya Kato

Chiba University 


\section{Research Article}

\section{Keywords:}

Posted Date: March 3rd, 2022

DOI: https://doi.org/10.21203/rs.3.rs-1379303/v1

License: (c) (1) This work is licensed under a Creative Commons Attribution 4.0 International License. Read Full License 


\section{Abstract}

This pilot study aimed to investigate the utility of texture and color enhancement imaging (TXI) with magnified endoscopy (ME) for the preoperative diagnosis of superficial nonampullary duodenal epithelial tumors (SNADETs). We prospectively evaluated 12 SNADETs. The visibility for ME-TXI, ME with indigo carmine (ICME) - white-light imaging (WLI), ICME-TXI compared to ME-NBI (narrow-band imaging) was scored ( +2 to -2 ME-NBI was set as score 0 ) by 3 experts. Scores +2 and +1 were defined as improved visibility. The interobserver agreement for improved visibility of surface structure (SS) was evaluated. Sensitivity, specificity, and positive predictive value (PPV) for Vienna Classification (VCL) C4/5 associated with the preoperative diagnosis of ICME-TXI were analyzed. The SS visibility score of ICME-TXI was significantly higher than that of ME-NBI, ME-TXI, and ICME-WLI ( $P<0.001$ respectively). The kappa coefficients of reliability for interobserver agreement for the SS visibility improvement with ICME-TXI were $0.70,0.96$, and 0.96 . All endoscopists preferred ICME-TXI for visualizing SS mostly for all lesions. The sensitivity, specificity, and PPV (\%) of ICME-TXI for VCL C4/5 were 80, 66.7, and 63.2, respectively. ICMETXI facilitates the visibility of the SS of SNADETs and may contribute to their preoperative diagnosis.

\section{Introduction}

The incidence of identified superficial nonampullary duodenal epithelial tumors (SNADETs) has gradually increased owing to advancements in endoscopic technology [1]. Pancreaticoduodenectomy (PD) is a standard treatment for duodenal cancers. However, PD is an invasive treatment with a mortality rate ranging from $1 \%-4 \%[2,3]$. Therefore, it is very important to accurately diagnose SNADETs to allow the less invasive management such as underwater endoscopic mucosal resection [4-6] or endoscopic submucosal dissection [7-10].

The accuracy of preoperative biopsy for SNADETs is unsatisfactory, ranging from $68-71.6 \%[11,12]$. In addition, small biopsy bites for SNADETs can cause severe fibrosis, which may become later obstacles for endoscopic resection [11]. Nowadays, there are more studies of the utility of image-enhanced endoscopy (IEE) for SNADETs. Magnified endoscopy (ME) with narrow-band imaging (NBI) for SNADETs is useful [13-15]. Further, the accuracy of diagnosis for the Vienna Classification (VCL) C3, C4 or C3, $\mathrm{C} 4 / 5[16,17]$ has been reported to range from $65.1-87 \%$ [13-15], suggesting that endoscopic diagnosis is at least comparable to biopsy.

In 2020, Olympus Medical Systems Corporation (Tokyo, Japan) introduced a new IEE system for texture and color enhancement imaging (TXI). In TXI, an image usually obtained by white-light irradiation is divided into a texture image and a base image. After enhancing the texture and correcting the color tone and brightness, the images are combined [18]. We previously reported the usefulness of TXI for visualizing gastric mucosal atrophy and gastric neoplasms [18]. However, its utility with ME for SNADETs has not yet been clarified. 
Thus, this pilot study aimed to investigate the utility of TXI with ME for the preoperative diagnosis of SNADETS.

\section{Methods}

\section{Study design and participants}

This prospective study was conducted at Chiba University Hospital (Japan) between March and July 2021. Patients diagnosed with sporadic SNADETs were prospectively enrolled in this study before diagnostic endoscopy. After endoscopic diagnosis, all patients underwent endoscopic resection or an operation. This study was reviewed and approved by the institutional review board of Chiba University School of Medicine and was registered at the University Hospital Medical Information Network (UMIN000041436). Informed consent was obtained from all partcipants. All methods were performed in accordance with the relevant guidelines and regulations.

\section{Instruments}

We used the CV-1500 light source equipped with a TXI system and the GIF-XZ1200 and GIF-H290Z endoscopes (Olympus Medical Systems Corporation, Tokyo, Japan). There are two types of TXI: mode 1 and mode 2. In TXI mode 1, the color tone changes are more coordinated than in TXI mode 2. In this study, we used only TXI mode 1. For the structure enhanced mode, "A7" was selected for NBI and (whitelight imaging) WLI, while "strong" was selected for TXI [18].

\section{Magnified endoscopy}

ME with WLI (ME-WLI), NBI (ME-NBI), and ME with TXI (ME-TXI) were performed for each lesion. For MEWLI and ME-TXI, indigo carmine (IC) dye was also used (ICME-WLI, ICME-TXI, respectively). During the preoperative endoscopic diagnosis of SNADETs, the lesions were assessed for size $(\mathrm{mm})$, location in the duodenum, macroscopic findings, endoscopic diagnosis according to VCL [16, 17], and the deposition of white opaque substance (WOS) [19]. The macroscopic findings were assessed according to the Paris endoscopic classification [20]. VCL endoscopic diagnosis was based on the presence of WOS, size of the lesion, and SS (closed- or open-loop) as described in a previous report [15]. Closed-loop structures were defined as oval-shaped mucosal structures that could be interpreted as connecting the starting point of the mucosa to the endpoint. Open-loop structures were defined as linear mucosal structures [15]. Endoscopic diagnosis with ICME-TXI was performed separated by each endoscopist.

\section{Evaluation of pathology}

All the tissue samples were sent for histological analysis with standard hematoxylin and eosin stain and were evaluated by gastrointestinal pathologists. The pathological diagnosis was according to the VCL, with classification into VCL C3 and C4/5. The details of VCL C4/5 are as follows; high-grade adenoma (C4.1), noninvasive carcinoma (C4.2), suspicious for invasive carcinoma (C4.3), intramucosal carcinoma (C5.1), and submucosal carcinoma (C5.2). 


\section{Outcomes}

The primary endpoint of this study was the visibility of the SS for SNADETs with ICME-TXI. We also assessed the visibility of the SNADETs' blood vessels. The visibility for ME-TXI, ICME-WLI, ICME-TXI compared to ME-NBI (ME-NBI was set as score 0 ) was scored by three experts (an endoscopist with > 5 years of IEE experience) in five stages, a modification of previous reports [21-23]. The details of the score are as follows, +2 (improved visibility remarkably), +1 (improved visibility), 0 (unchanged visibility), - 1 (worsened visibility), -2 (worsened visibility remarkably). Scores +2 and +1 were defined as improved visibility. The interobserver agreements for the improved visibility of SS were evaluated. In addition, the favored modality by the examiners for the evaluation of the SS and blood vessels was assessed. The sensitivity, specificity, and positive predictive value (PPV) for VCL C4/5 associated with the preoperative diagnosis of ICME-TXI and preoperative biopsy were also investigated.

\section{Sample size calculation}

There were no data regarding ICME-TXI for SNADETs. As this was a pilot study, 12 lesions were enrolled based on criteria from a previous report [24].

\section{Statistical analysis}

Baseline data were presented as mean \pm standard deviation (SD). The differences in visibility scores were analyzed using Friedman's test with Bonferroni correction. Interobserver agreement for the visibility improvement was assessed with Cohen's Kappa analysis. The kappa coefficient of reliability was classified as follows: $0.0-0.2$ (slight agreement), $0.21-0.40$ (fair agreement), $0.41-0.60$, (moderate agreement), $0.61-0.80$ (substantial agreement), and 0.81-1.0 (almost perfect or perfect agreement). All statistical analyses were performed using the Statistical Package for the Social Sciences software version 26 (SPSS Inc., Chicago, IL, USA). P-values $<0.05$ were considered statistically significant.

\section{Results}

\section{Patients and lesions}

In total, 11 patients and 12 lesions were evaluated. The characteristics of the patients and lesions are shown in Table 1. The median tumor size was $10 \mathrm{~mm}$. The macroscopic findings were lla dominant. Seven lesions (58.3\%) were VCL C3, and 5 lesions (41.7\%) were VCL C4/5. WOS was identified in 8 lesions (66.7\%). Representative cases of endoscopic observation in each modality for SNADETs are shown in Figs. 1a and 1b. 
Table 1

Characteristics of the participants and lesions

\begin{tabular}{|ll|}
\hline & All patients \\
\hline Age (years), median (range) & $(\mathrm{n}=11)$ \\
\hline Male/female & $69(37-80)$ \\
\hline & $5 / 6$ \\
\hline Size (mm), median (range) & All lesions \\
\hline Location of the lesions, $n$ (\%) & $(\mathrm{n}=12)$ \\
\hline 2nd (oral side of the major papilla) & $10(3-100)$ \\
\hline 2nd (anal side of the major papilla) & \\
\hline Macroscopic findings, $n$ (\%) & $8(66.7)$ \\
\hline Is & $4(33.3)$ \\
\hline Ila & $2(16.7)$ \\
\hline Ilc & $9(75.0)$ \\
\hline Histopathological diagnosis, $n$ (\%) & $1(8.3)$ \\
\hline VCL C3 & \\
\hline VCL C4/5 & $7(50.0)$ \\
\hline Presence of WOS, $n$ (\%) & $7(58.3)$ \\
\hline Resection, $n$ (\%) & $5(41.7)$ \\
\hline CSP & $8(66.7)$ \\
\hline VEMR & \\
\hline resection & $4(33.3)$ \\
\hline
\end{tabular}


Table 2

a. P-value of visibility scores for surface structure.

\begin{tabular}{|c|c|c|c|c|c|}
\hline \multicolumn{6}{|l|}{ P-value } \\
\hline $\begin{array}{l}\text { ME-NBI vs } \\
\text { ME-TXI }\end{array}$ & $\begin{array}{l}\text { ME-NBI vs } \\
\text { ICME-WLI }\end{array}$ & $\begin{array}{l}\text { ME-NBI vs } \\
\text { ICME-TXI }\end{array}$ & $\begin{array}{l}\text { ME-TXI vs } \\
\text { ICME-WLI }\end{array}$ & $\begin{array}{l}\text { ME-TXI vs } \\
\text { ICME-TXI }\end{array}$ & $\begin{array}{l}\text { ICME-WLI vs } \\
\text { ICME-TXI }\end{array}$ \\
\hline 1.000 & 1.000 & $<0.001$ & 1.000 & $<0.001$ & $<0.001$ \\
\hline
\end{tabular}

\section{Visibility score}

The mean visibility scores ( \pm SD) in each modality are shown in Figs. $2 a$ and $2 b$. As described previously, the mean visibility score of ME-NBI was set as 0 . For visibility of SS, the mean visibility scores for ME-TXI, ICME-WLI, ICME-TXI were $-0.08 \pm 0.81,0.22 \pm 0.87,1.58 \pm 0.60$, respectively. For the visibility score of blood vessels, the mean visibility score for ME-TXI, ICME-WLI, ICME-TXI were $-0.78 \pm 0.80,-1.19 \pm 0.71$, $-0.58 \pm 0.55$, respectively. The P-value of the visibility scores of SNADETs in ME-TXI, ICME-WLI, and ICME-TXI compared to ME-NBI are shown in Tables 2A and 2B. In summary, the visibility score of SS in ICME-TXI was significantly higher than those of ME-NBI, ME-TXI, and ICME-WLI ( $P<0.001$, respectively). The visibility scores of blood vessels in ME-TXI, ICME-WLI, ICME-TXI were significantly lower than ME-NBI $(P=0.001, P<0.001, P=0.028$, respectively). In addition, the visibility score of blood vessels in ICME-TXI was significantly higher than ICME-WLI $(P=0.003)$.

Preference of modality for surface structure and blood vessels.

For visualizing SS, all endoscopists preferred ICME-TXI mostly for all lesions. On the other hand, for visualizing blood vessels, all endoscopists preferred ME-NBI mostly for all lesions.

Interobserver agreement for the improvement of visibility for surface structure with ICME-TXI.

The kappa coefficients of reliability for interobserver agreement for the visibility improvement for SS with ICME-TXI were $0.70,0.96$, and 0.96 . Interobserver agreements for SNADETs were "substantial agreement" or "almost perfect or perfect agreement."

Sensitivity and PPV for VCL C4/5 in association with preoperative diagnosis of ICME-TXI.

The sensitivity, specificity, and PPV (\%) for VCL C4/5 associated with a preoperative diagnosis of ICMETXI (calculated for 36 lesions, 12 for each endoscopist) were 80, 66.7, and 63.2, respectively. Preoperative biopsy was performed in 9 of 12 lesions. The sensitivity, specificity, and PPV (\%) for VCL C4/5 in preoperative biopsy were $40.0,75.0$, and 66.7 , respectively. The sensitivity of preoperative diagnosis with ICME-TXI for SNADETs tended to be higher than with preoperative biopsy.

\section{Discussion}

This study is the first to report the usefulness of ME-TXI, including ICME-TXI. Conventionally, NBI was the main modality for ME [13-15]. 
The visibility of the SS with ICME-WLI and ME-TXI did not differ from that of ME-NBI. But with ICME-TXI, the SS were more visible than with ME-NBI. The visibility of the SS did not improve with IC or texture enhancement of TXI alone. It is thought that accumulated IC in the concave parts of the SNADETs' surface made the ductal structure outline stand out due to the color emphasis. This made the SS easy to recognize. The kappa coefficients of reliability for interobserver agreement for improving visibility for SS of ICME-TXI and the percentage for endoscopists' preference for ICME-TXI regarding the recognition of the SS were high. It seems that the reproducibility is high. Akazawa et al. reported that SNADETs showed distinct endoscopic features according to the mucin phenotype [25]. For instance, the gastric phenotype had dense pattern dilatation of the intervening part with ME-NBI [25]. Although this study did not analyze the mucin phenotype, it could be possible to distinguish mucus phenotype preoperatively by observing the SS with ICME-TXI.

In the previous report of the ME-NBI diagnosis of SNADETs referred to for the diagnosis in this study, the sensitivity, specificity for VCL C4/5 in association with the preoperative diagnosis were $90.5 \%$ and $52.4 \%$ respectively [15], which were comparable with our results. The sensitivity of preoperative diagnosis for ICME-TXI tended to be superior to that of biopsy in this study. ICME-TXI can recognize the SS with ease, and preoperative diagnosis may be made more efficiently.

$\mathrm{NBI}$ has an absorption range wavelength that matches the blood vessels. Capillaries in the superficial mucosal layer are emphasized by the $415 \mathrm{~nm}$ light and are displayed in brown, whereas deeper mucosal and submucosal vessels are visualized by the $540 \mathrm{~nm}$ light and are displayed in cyan [26]. Therefore, ME$\mathrm{NBI}$ was still superior in vascular visibility to any other modality in this study. Interestingly, visibility scores for blood vessels of ME-TXI tended to be higher than ICME-WLI. In addition, those of ICME-TXI were significantly higher than with ICME-WLI. The outline of the blood vessel is emphasized by the texture enhancement of TXI and contrast with IC as well, so the blood vessels were easier to identify than with ICME-WLI. The diagnostic method used in this study does not include evaluating blood vessels, but TXI may be useful for diagnosis including assessing the vascular structure.

This study had several limitations. First, it was a pilot study with a sample of only 12 . Therefore, the results of this study only suggested the potential of ICME-TXI in the preoperative diagnosis of SNADETs. Second, the diagnostic method referred to in this study was originally for ME-NBI. A large prospective study using a specific method for TXI for the preoperative diagnosis of SNADETs is crucial for assessing the true value of ICME-TXI.

In conclusion, ICME-TXI facilitates the visibility of the SS of SNADETs and may contribute to the preoperative diagnosis of SNADETs.

\section{Declarations}

Author Contributions: Designed the study, conducted the experiment, collected, analyzed, and interpreted data, wrote the manuscript: KO, TM, DM; designed the study, analyzed and interpreted data, assisted in 
writing the manuscript: AK, WS, AN, TI, TK, KK, NA, YO, TT, KS, JK, NK. All authors approved the final version of the manuscript.

\section{Data availability statement}

The datasets generated and/or analyzed during the current study are not publicly available due to the protection of personal information.

\section{References}

1. Yamasaki, Y. et al. Differentiation between duodenal neoplasms and non-neoplasms using magnifying narrow-band imaging - Do we still need biopsies for duodenal lesions? Dig. Endosc. 32, 84-95 (2020).

2. Yeo, C. J. et al. Six hundred fifty consecutive pancreaticoduodenectomies in the 1990s: pathology, complications, and outcomes. Ann. Surg. 226, 248-57; discussion 257 (1997).

3. Cameron, J. L., Riall, T. S., Coleman, J. \& Belcher, K. A. One thousand consecutive pancreaticoduodenectomies. Ann. Surg. 244, 10-15 (2006).

4. Binmoeller, K. F., Shah, J. N., Bhat, Y. M. \& Kane, S. D. 'Underwater' EMR of sporadic laterally spreading nonampullary duodenal adenomas (with video). Gastrointest. Endosc. 78, 496-502 (2013).

5. Yamasaki, Y. et al. Underwater endoscopic mucosal resection for superficial nonampullary duodenal adenomas. Endoscopy 50, 154-158 (2018).

6. Okimoto, K. et al. Utility of underwater EMR for nonpolypoid superficial nonampullary duodenal epithelial tumors $\leq 20 \mathrm{~mm}$. Gastrointest. Endosc. 95, 140-148 (2022).

7. Yamamoto, Y., Yoshizawa, N., Tomida, Het al., Fujisaki, J. \& Igarashi, M. Therapeutic outcomes of endoscopic resection for superficial non-ampullary duodenal tumor. Dig. Endosc. 26, Suppl 2, 50-56 (2014).

8. Jung, J. H. et al. Endoscopic submucosal dissection for sessile, nonampullary duodenal adenomas. Endoscopy 45, 133-135 (2013).

9. Matsumoto, S., Miyatani, H. \& Yoshida, Y. Endoscopic submucosal dissection for duodenal tumors: a single-center experience. Endoscopy 45, 136-137 (2013).

10. Hoteya, S. et al. Endoscopic submucosal dissection for nonampullary large superficial adenocarcinoma/adenoma of the duodenum: feasibility and long-term outcomes. Endosc. Int. Open 1, 2-7 (2013).

11. Kinoshita, S. et al. Accuracy of biopsy for the preoperative diagnosis of superficial nonampullary duodenal adenocarcinoma. Gastrointest. Endosc. 86, 329-332 (2017). 
12. Goda, K. et al. Endoscopic diagnosis of superficial non-ampullary duodenal epithelial tumors in Japan: multicenter case series. Dig. Endosc. 26, Suppl 2, 23-29 (2014).

13. Mizumoto, T. et al. Clinical usefulness of magnifying endoscopy for non-ampullary duodenal tumors. Endosc. Int. Open 5, E297-E302 (2017).

14. Kakushima, N. et al. Magnified endoscopy with narrow-band imaging for the differential diagnosis of superficial non-ampullary duodenal epithelial tumors. Scand. J. Gastroenterol. 54, 128-134 (2019).

15. Nakayama, A. et al. How I do it: endoscopic diagnosis for superficial non-ampullary duodenal epithelial tumors. Dig. Endosc. 32, 417-424 (2020).

16. Dixon, M. F. Gastrointestinal epithelial neoplasia: Vienna revisited. Gut 51, 130-131 (2002).

17. Stolte, M. The new Vienna classification of epithelial neoplasia of the gastrointestinal tract: advantages and disadvantages. Virchows Arch. 442, 99-106 (2003).

18. Ishikawa, T. et al. Efficacy of Texture and Color Enhancement Imaging in visualizing gastric mucosal atrophy and gastric neoplasms. Sci. Rep. 11, 6910 (2021).

19. Yoshimura, N. et al. Endoscopic features of nonampullary duodenal tumors with narrow-band imaging. Hepato-Gastroenterology 57, 462-467 (2010).

20. Participants in the Paris workshop. The Paris endoscopic classification of superficial neoplastic lesions: esophagus, stomach, and colon. Gastrointest. Endosc. 58 Suppl (2003)

21. Yoshifuku, Y. et al. Evaluation of the visibilityof early gastric cancer using linked color imaging and blue laser imaging. BMC Gastroenterol. 17, 150 (2017).

22. Ninomiya, Y. et al. Clinical impact of dual red imaging in colorectal endoscopic submucosal dissection: a pilot study. Therap. Adv. Gastroenterol. 9, 679-683 (2016).

23. Nakamura, K. et al. Usefulness of linked color imaging in the early detection of superficial esophageal squamous cell carcinomas. Esophagus 18, 118-124 (2021).

24. Julious, S. A. Sample size of 12 per group rule of thumb for a pilot study. Pharmaceut. Statist. 4, 287-291 (2005).

25. Akazawa, Y. et al. Endoscopic and Clinicopathological Features of Superficial Non-Ampullary Duodenal Tumor Based on the Mucin Phenotypes. Digestion 102, 663-670 (2021).

26. Muto, M. et al. Narrow-band imaging of the gastrointestinal tract. J. Gastroenterol. 44, 13-25 (2009).

\section{Figures}

\section{Figure 1}

\section{Representative cases of SNADETs for each modality.}


SNADET, Superficial nonampullary duodenal epithelial tumors; ME-WLI, magnified endoscopy with whitelight imaging, ME-NBI, magnified endoscopy with narrow-band imaging; ME-TXI, magnified endoscopy with texture and color enhancement imaging; ICME-WLI, indigo carmine dye with ME-WLI; ICME-TXI, indigocarmine dye with ME-TXI; VCL, Vienna classification; IMC, intramucosal carcinoma

\section{(a) $13 \mathrm{~mm}$, lla, pathological diagnosis of VCL C3.}
A. ME-NBI
B. ME-TXI
C. ICME-WLI
D. ICME-TXI. Open-loop was observed.

(b) $10 \mathrm{~mm}$, lla, pathological diagnosis of VCL C4/5 (IMC).
A. ME-NBI
B. ME-TXI
C. ICME-WLI
D. ICME-TXI. Closed-loop was observed.

\section{Figure 2}

\section{The mean visibility scores (described mean \pm SD) for each modality}

The mean visibility score of ME-NBI was set as score 0 .

SD, standard deviation; ME-WLI, magnified endoscopy with white-light imaging; ME-NBI, magnified endoscopy with narrow-band imaging; ME-TXI, magnified endoscopy with texture and color enhancement imaging; ICME-WLI, indigo carmine dye with ME-WLI; ICME-TXI, indigocarmine dye with ME-TXI 